\title{
Circadian oscillations within the hippocampus support memory formation and persistence
}

\author{
Kristin L. Eckel-Mahan* \\ Sassone-Corsi Lab, Center for Epigenetics and Metabolism, Department of Biological Chemistry, University of California, Irvine, Irvine, CA, USA
}

\section{Edited by:}

Joerg H. Stehle, Goethe University

Frankfurt, Germany

\section{Reviewed by:}

Urs Albrecht, University of Fribourg, Switzerland

Erik Maronde, University of Frankfurt, Germany

\section{*Correspondence:}

Kristin L. Eckel-Mahan, Sassone-Corsi Lab, Center for Epigenetics and Metabolism, Department of Biological Chemistry, University of California, Irvine, Irvine, CA, USA.

e-mail:mahank@uci.edu
The ability to sustain memories over long periods of time, sometimes even a lifetime, is one of the most remarkable properties of the brain. Much knowledge has been gained over the past few decades regarding the molecular correlates of memory formation. Once a memory is forged, however, the molecular events that provide permanence are as of yet unclear. Studies in multiple organisms have revealed that circadian rhythmicity is important for the formation, stability, and recall of memories (Gerstner et al., 2009). The neuronal events that provide this link need to be explored further. This article will discuss the findings related to the circadian regulation of memory-dependent processes in the hippocampus. Specifically, the circadian-controlled mitogen-activated protein kinase (MAPK) and cAMP signal transduction pathway plays critical roles in the consolidation of hippocampus-dependent memory. A series of studies have revealed the circadian oscillation of this pathway within the hippocampus, an activity that is absent in memory-deficient, transgenic mice lacking $\mathrm{Ca}^{2+}$-stimulated adenylyl cyclases. Interference with these oscillations proceeding the cellular memory consolidation period impairs the persistence of hippocampus-dependent memory. These data suggest that the persistence of long-term memories may depend upon reactivation of this signal transduction pathway in the hippocampus during the circadian cycle. New data reveals the dependence of hippocampal oscillation in MAPK activity on the suprachiasmatic nucleus, again underscoring the importance of this region in maintaining the circadian physiology of memory. Finally, the downstream ramification of these oscillations in terms of gene expression and epigenetics should be considered, as emerging evidence is pointing strongly to a circadian link between epigenetics and long-term synaptic plasticity.

Keywords: circadian, hippocampus, memory, mitogen-activated protein kinase, adenylyl cyclase

\section{INTRODUCTION}

Circadian rhythms are oscillations that occur with approximately 24-h periodicity. These rhythms persist throughout the body and play essential roles in physiology. Single cell oscillators interact within specific tissues to drive tissue-specific activities, which in turn provide information for the rest of the body (Schibler and Sassone-Corsi, 2002). Neurons of the central nervous system are among the single cell oscillators in the body and these coordinately function within specific brain regions to direct activities as disparate as melatonin secretion, thermoregulation, and memory formation and maintenance. As a region devoted to short-term to long-term memory conversion, the hippocampus provides a pivotal node for the intersection of circadian rhythms and memory formation.

The circadian clock is organized hierarchically. The suprachiasmatic nucleus $(\mathrm{SCN})$ of the brain provides synchronization of circadian clocks throughout the body and lesions of the small bilateral nuclei that make up this region produce molecular and behavioral arrhythmicity in mammals (Stephan and Zucker, 1972; Eastman et al., 1984). Some CNS tissues including the pineal gland and the hypothalamus host robust SCN-driven oscillations, resulting in the rhythmic release of melatonin, for example. More recent studies have focused on rhythmicity in other CNS tissues, including those involved in memory processing and cognition. The expression and oscillation of various circadian clock genes has been noted in cells of the cortex, basolateral and central amygdala, olfactory bulb, and the hippocampus, among other CNS regions (Jilg et al., 2005, 2010; Prolo et al., 2005; Granados-Fuentes et al., 2006; Eckel-Mahan et al., 2008; Pantazopoulos et al., 2011; Phan et al., 2011).

Surprisingly little is clear, however, concerning the ramifications of the presence of the circadian clock on synaptic plasticity in distinct regions of the forebrain. With more precise in vivo recording technologies, more attention is being paid to the idea that neuronal synaptic plasticity, and particularly, firing rates themselves, may be modulated by the circadian clock. Several studies now show that the magnitude of hippocampal LTP varies based on the time of day. Specifically, when population spikes were measured following hippocampal Schaffer collateral stimulation, the degree of population spike enhancement was much greater during LTP in slices prepared at night at opposed to the day (Chaudhury et al., 2005). Interestingly, this was true of strains of mice that rhythmically secrete melatonin as well as those that are deficient in melatonin production. Furthermore, recordings from hippocampal pyramidal cells of the CA1 region demonstrate that the basal firing rates are not static over time but rather, when measured 


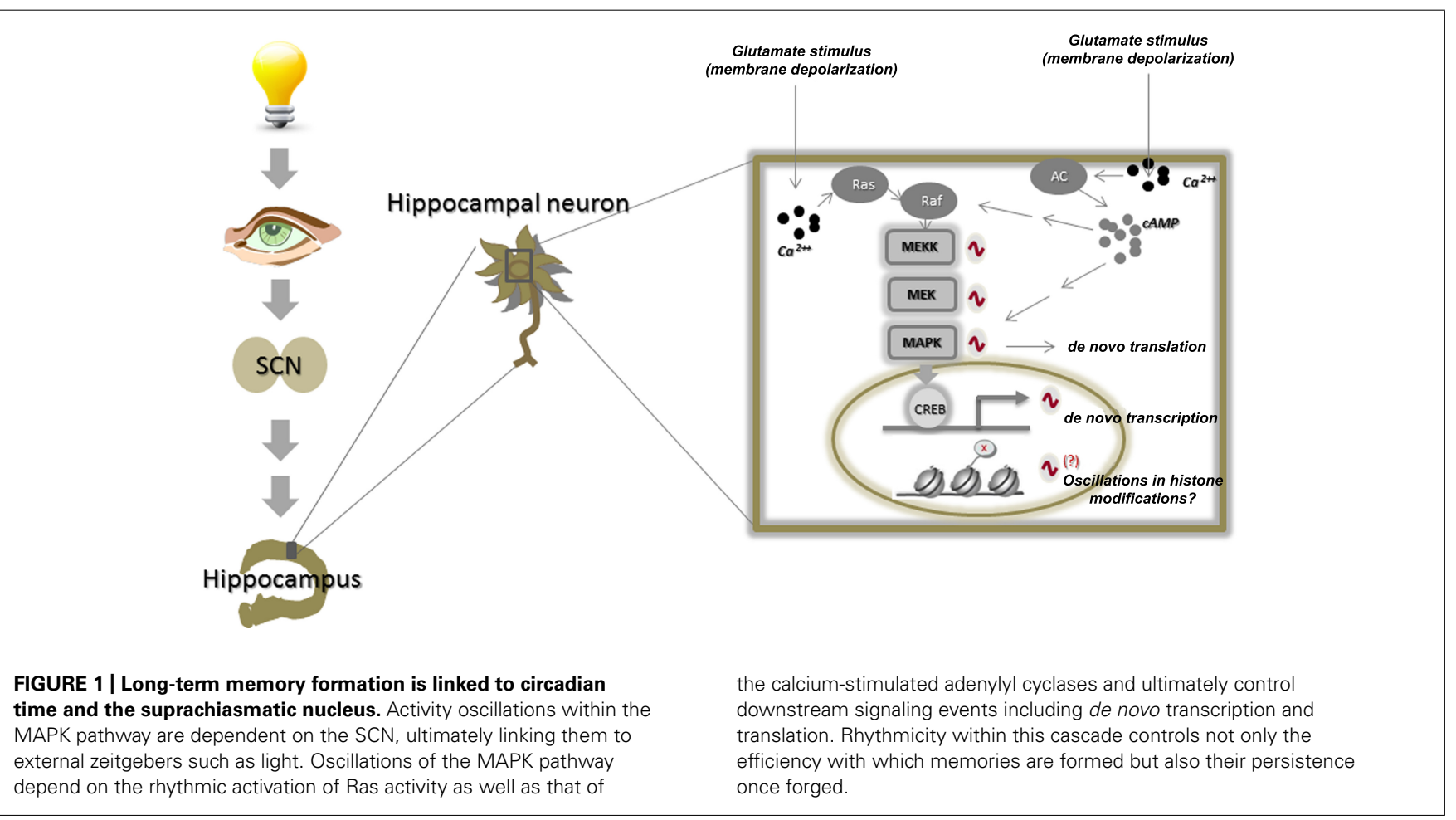

for many hours in vivo, exhibit firing rates of a sinusoidal nature and with a 25-h period (Munn and Bilkey, 2011). Fluctuations in synaptic plasticity that depend on diurnal luminescence have also been observed in the visual cortex. The induction of LTP and LTD in areas $2 / 3$ of the primary visual cortex after stimulation of the dorsal lateral geniculate nucleus shows a strong dependence on the lighting cycle. Interestingly, while low frequency stimulation of $3 \mathrm{~Hz}$ can generate LTD in light-exposed animals, the same stimulus generates LTP in dark-exposed animals (Tsanov and Manahan-Vaughan, 2007). While the idea that synaptic plasticity in the visual cortex would be affected by circadian fluctuations in lighting may seem obvious, it provides an example of just how disparate neuronal responses can be - even after the same stimulus - depending on the basal firing state of the integrating network.

\section{DEPENDENCE OF CIRCADIAN RHYTHMICITY AND HIPPOCAMPAL LONG-TERM MEMORY ON MAPK ACTIVATION}

While the mechanisms underlying the circadian effects on LTP are still being explored, some pathways implicated in synaptic plasticity, particularly those associated with hippocampal potentiation, have been explored in detail. Among the singling events known to be important for the induction of hippocampal LTP is the activation of the mitogen-activated protein kinase (MAPK) cascade. Activation of this pathway is necessary for both the transcriptional and the translational events underlying long-term memory formation in the hippocampus (Figure 1) (Atkins et al., 1998; Davis et al., 2000; Kelleher III et al., 2004). The MAPK cascade encompasses important proteins in signal transduction. The MAPKs are highly conserved across all eukaryotes and their roles extend beyond the cytoplasm to the nucleus, where they can directly modulate gene transcription. The MAPK cascade generally consists of three tiered kinase proteins. The kinase upstream of MAPK is typically referred to as the MAPK kinase, MKK, or MEK protein. These kinases lie downstream of a third activator kinase, the MAPK kinase kinase, MEK kinase, or MKKs. Five MAPK cascades have been identified including the ERK1/2, JNK, p38, ERK5, and the atypical MAPK proteins which include ERK3 and ERK4 among others (reviewed in Gaestel, 2008). The ERK1/2 pathway is well known to regulate cell differentiation and growth while the JNK and p38 pathways are involved in stress response (reviewed in Schaeffer and Weber, 1999). Several mitogens and growth factors are known to activate these pathways. In neurons (unlike many other cell types), however, the activation of calcium-sensitive adenylyl cyclases appears to be important for the transduction of membrane stimulation to activation of the MAPK cascade (Impey et al., 1998; Dugan et al., 1999). While the contribution of MAPK to memory consolidation is largely based on the administration of inhibitors (which are often dirty and impair the function of additional relevant pathways), the administration of MAPK antisense oligonucleotides in area CA1 of the hippocampus underscores the importance of this kinase in hippocampal plasticity (Wu et al., 1999).

The role of the MAPK pathway in circadian rhythmicity is beginning to be established. The p442/44 MAPK pathway is light responsive in neurons of the rodent SCN (Obrietan et al., 1998; Butcher et al., 2002) and several MAPK isoforms have been implicated in cellular timekeeping in a variety of other organisms (de Paula et al., 2008). While cAMP levels are antagonistic to MAPK phosphorylation and activation in some cell types, in neurons the activation of the MAPK is tightly coupled to increases in cAMP. This coupling appears to be important for clock time 
keeping in the brain. Circadian oscillations in cAMP are essential modulators of SCN rhythmicity and infusion of adenylate cyclase agonists or antagonists into the third ventricle can alter the phase of rhythmic gene expression in the SCN (O'Neill et al., 2008). cAMP has also been observed as oscillatory in the hippocampus where its reliance on the calcium-sensitive adenylate cyclases seems to be critical for its oscillatory presence (EckelMahan et al., 2008).

\section{DEPENDENCE OF HIPPOCAMPAL LONG-TERM MEMORY FORMATION AND PERSISTENCE ON NORMAL CIRCADIAN RHYTHMICITY}

Recent work centered on the role of hippocampal P-ERK oscillations has revealed that hippocampal oscillations are actually maintained by the SCN. While sham lesions of the SCN produce no changes in the rhythmicity by which the hippocampal MAPK pathway is activated, electrolytic lesions of the SCN ablate circadian oscillations in this pathway's activation. Long-term memory deficits are also incurred by such lesions (Phan et al., 2011). The control of hippocampal MAPK oscillations by the SCN demonstrates the tight links between the pacemaker and memory formation. As some oscillations can persist independently of the SCN, or can be entrained by alternative zeitgebers to light (such as food), the fact that SCN lesions impair oscillations in hippocampal MAPK activity indicates that hippocampal circadian oscillations at least in this signaling cascade, are not intrinsic to this region, and rather rely on a network of neurons that provide circadian information from the SCN. This is supported by the idea that constant light ablates circadian fluctuations in hippocampal MAPK activity (Eckel-Mahan et al., 2008). What remains to be seen is whether memory training can function as a zeitgeber for the hippocampus and other memory-associated areas such as the cortex and the amygdala. For example, in the liver feeding restriction can restore rhythmicity to a liver rendered arrhythmic by loss of SCN rhythmicity (Damiola et al., 2000; Stokkan et al., 2001). Are there temporal windows at which memory training might compromise the synchronous nature of circadian communication from the

\section{REFERENCES}

Angeles-Castellanos, M., Amaya, J. M., Salgado-Delgado, R., Buijs, R. M., and Escobar, C. (2011). Scheduled food hastens re-entrainment more than melatonin does after a 6-h phase advance of the light-dark cycle in rats. J. Biol. Rhythms 26, 324-334.

Atkins, C. M., Selcher, J. C., Petraitis, J. J., Trzaskos, J. M., and Sweatt, J. D. (1998). The MAPK cascade is required for mammalian associative learning. Nat. Neurosci. 1, 602-609.

Butcher, G. Q., Doner, J., Dziema, H., Collamore, M., Burgoon, P. W., and Obrietan, K. (2002). The p42/44 mitogen-activated protein kinase pathway couples photic input to circadian clock entrainment. J. Biol. Chem. 277, 29519-29525.

Chaudhury, D., Wang, L. M., and Colwell, C. S. (2005). Circadian regulation of hippocampal long-term potentiation. J. Biol. Rhythms 20, 225-236.

Damiola, F., Le Minh, N., Preitner, N., Kornmann, B., Fleury-Olela, F., and Schibler, U. (2000). Restricted feeding uncouples circadian oscillators in peripheral tissues from the central pacemaker in the suprachiasmatic nucleus. Genes Dev. 14, 2950-2961.

Davis, S., Vanhoutte, P., Pages, C., Caboche, J., and Laroche, S. (2000). The MAPK/ERK cascade targets both Elk-1 and cAMP response elementbinding protein to control long-term potentiation-dependent gene expression in the dentate gyrus in vivo. $J$. Neurosci. 20, 4563-4572.

de Paula, R. M., Lamb, T. M., Bennett, L., and Bell-Pedersen, D. (2008). A connection between MAPK pathways and circadian clocks. Cell Cycle 7, 2630-2634.

SCN to the hippocampus? What about situations in which circadian rhythms are involuntarily disrupted? In the event that cross time zone travel or other inopportune (from a circadian standpoint) events render one's rhythms disrupted, are there training paradigms or schedules that we might employ to overcome the deficit that this may cause on CNS regions involved in learning and memory, such as the hippocampus? Such behavioral approaches may not be so farfetched considering the similarities by which zeitgebers entrain other tissues. A striking example of a zeitgeber overcoming circadian rhythm loss has been noted recently. In genetically arrhythmic animals, the restriction of food to a small time window restores rhythmicity in vivo. Rhythms induced by food availability can even override the rhythmicity imposed by the light/dark cycle and interestingly, can accelerate reentrainment speed after phase shifting in rodents (Angeles-Castellanos et al., 2011). These data implicate a non-SCN food-entrainable oscillator that in isolation can compensate for the loss of SCN rhythmicity while in concert with the SCN, keeps the body ticking normally during periods of ample nutrient availability. Such an override of local clocks in the brain seems achievable and may be an avenue worthy of consideration for individuals that suffer from regular circadian disturbance.

Becoming increasingly clear is that baseline synaptic plasticity is not a static phenomenon but rather changes over time with the circadian cycle. These changes are dependent on the time of day - as discussed here - but also on the previous state of sleep and wakefulness in an organism (Vyazovskiy et al., 2008; Gilestro et al., 2009). Such changes in synaptic plasticity ultimately rely on intracellular fluctuations in small molecules (such as cAMP and calcium), circadian changes in enzyme activity (i.e., adenylyl cyclase, and MAPK activity), and circadian changes in gene transcription. As circadian oscillating events, such changes are essential modifiers of memory formation and maintenance. As the concept that synaptic plasticity and therefore cognition are greatly influenced by the circadian clock has become empirically validated, it becomes necessary to address the role of circadian rhythmicity in behavioral study design.

Dugan, L. L., Kim, J. S., Zhang, Y., Bart, R. D., Sun, Y., Holtzman, D. M., and Gutmann, D. H. (1999). Differential effects of cAMP in neurons and astrocytes. Role of B-raf. J. Biol. Chem. 274, 25842-25848.

Eastman, C. I., Mistlberger, R. E., and Rechtschaffen, A. (1984). Suprachiasmatic nuclei lesions eliminate circadian temperature and sleep rhythms in the rat. Physiol. Behav. 32, 357-368.

Eckel-Mahan, K. L., Phan, T., Han, S., Wang, H., Chan, G. C., Scheiner, Z. S., and Storm, D. R. (2008). Circadian oscillation of hippocampal MAPK activity and cAMP: implications for memory persistence. Nat. Neurosci. 11, 1074-1082.

Gaestel, M. (2008). Specificity of signaling from MAPKs to MAPKAPKs: kinases' tango nuevo. Front. Biosci. 13, 6050-6059.
Gerstner, J. R., Lyons, L. C., Wright, K. P. Jr., Loh, D. H., Rawashdeh, O., Eckel-Mahan, K. L., and Roman, G. W. (2009). Cycling behavior and memory formation. J. Neurosci. 29 , 12824-12830.

Gilestro, G. F., Tononi, G., and Cirelli, C. (2009). Widespread changes in synaptic markers as a function of sleep and wakefulness in Drosophila. Science 324, 109-112.

Granados-Fuentes, D., Tseng, A., and Herzog, E. D. (2006). A circadian clock in the olfactory bulb controls olfactory responsivity. J. Neurosci. 26 , 12219-12225.

Impey, S., Obrietan, K., Wong, S. T., Poser, S., Yano, S., Wayman, G., Deloulme, J. C., Chan, G., and Storm, D. R. (1998). Cross talk between ERK and PKA is required for $\mathrm{Ca}^{2+}$ stimulation of CREBdependent transcription and ERK 
nuclear translocation. Neuron 21, 869-883.

Jilg, A., Lesny, S., Peruzki, N., Schwegler, H., Selbach, O., Dehghani, F., and Stehle, J. H. (2010). Temporal dynamics of mouse hippocampal clock gene expression support memory processing. Hippocampus 20, 377-388.

Jilg, A., Moek, J., Weaver, D. R., Korf, H. W., Stehle, J. H., and von Gall, C. (2005). Rhythms in clock proteins in the mouse pars tuberalis depend on MT1 melatonin receptor signalling. Eur. J. Neurosci. 22, 2845-2854.

Kelleher, R. J. III, Govindarajan, A., Jung, H. Y., Kang, H., and Tonegawa, S. (2004). Translational control by MAPK signaling in long-term synaptic plasticity and memory. Cell 116, 467-479.

Munn, R. G., and Bilkey, D. K. (2011). The firing rate of hippocampal CAl place cells is modulated with a circadian period. Hippocampus. doi: 10.1002/hipo.20969

Obrietan, K., Impey, S., and Storm, D. R. (1998). Light and circadian rhythmicity regulate MAP kinase activation in the suprachiasmatic nuclei. Nat. Neurosci. 1, 693-700.

O’Neill, J. S., Maywood, E. S., Chesham, J. E., Takahashi, J. S., and Hastings, M. H. (2008). cAMP-dependent signaling as a core component of the mammalian circadian pacemaker. Science 320, 949-953.

Pantazopoulos, H., Dolatshad, H., and Davis, F. C. (2011). A fear-inducing odor alters PER2 and c-Fos expression in brain regions involved in fear memory. PLoS ONE 6, e20658. doi: 10.1371/journal.pone.0020658

Phan, T. X., Chan, G. C., Sindreu, C. B., Eckel-Mahan, K. L., and Storm, D. R. (2011). The diurnal oscillation of MAP (mitogen-activated protein) kinase and adenylyl cyclase activities in the hippocampus depends on the suprachiasmatic nucleus. J. Neurosci. 31, 10640-10647.

Prolo, L. M., Takahashi, J. S., and Herzog, E. D. (2005). Circadian rhythm generation and entrainment in astrocytes. J. Neurosci. 25, 404-408.

Schaeffer, H. J., and Weber, M. J. (1999). Mitogen-activated protein kinases: specific messages from ubiquitous messengers. Mol. Cell. Biol. 19, 2435-2444.

Schibler, U., and Sassone-Corsi, P. (2002). A web of circadian pacemakers. Cell 111, 919-922.

Stephan, F. K., and Zucker, I. (1972). Circadian rhythms in drinking behavior and locomotor activity of rats are eliminated by hypothalamic lesions. Proc. Natl. Acad. Sci. U.S.A. 69, 1583-1586.

Stokkan, K. A., Yamazaki, S., Tei, H. Sakaki, Y., and Menaker, M. (2001). Entrainment of the circadian clock in the liver by feeding. Science 291, 490-493.

Tsanov, M., and Manahan-Vaughan, D. (2007). The adult visual cortex expresses dynamic synaptic plasticity that is driven by the light/dark cycle. J. Neurosci. 27, 8414-8421.

Vyazovskiy, V. V., Cirelli, C., PfisterGenskow, M., Faraguna, U., and Tononi, G. (2008). Molecular and electrophysiological evidence for net synaptic potentiation in wake and depression in sleep. Nat. Neurosci. 11, 200-208.
Wu, S. P., Lu, K. T., Chang, W. C., and Gean, P. W. (1999). Involvement of mitogen-activated protein kinase in hippocampal long-term potentiation. J. Biomed. Sci. 6, 409-417.

Conflict of Interest Statement: The author declares that the research was conducted in the absence of any commercial or financial relationships that could be construed as a potential conflict of interest.

Received: 30 September 2011; accepted: 22 March 2012; published online: 17 April 2012.

Citation: Eckel-Mahan KL (2012) Circadian oscillations within the hippocampus support memory formation and persistence. Front. Mol. Neurosci. 5:46. doi: 10.3389/fnmol.2012.00046

Copyright (c) 2012 Eckel-Mahan. This is an open-access article distributed under the terms of the Creative Commons Attribution Non Commercial License, which permits non-commercial use, distribution, and reproduction in other forums, provided the original authors and source are credited. 